\title{
Combined Oocyte Retrieval and Robot-assisted Hysterectomy in a Super Morbidly Obese Patient with Endometrial Carcinoma
}

\author{
ESTHER H. CHUNG, PAULA C. BRADY, KIMBERLY KEEFE SMITH, MICHELLE R. DAVIS, \\ MICHAEL G. MUTO, RACHEL K. ASHBY and ELIZABETH S. GINSBURG \\ Department of Obstetrics, Gynecology, and Reproductive Biology, \\ Brigham and Women's Hospital and Harvard Medical School, Boston, MA, U.S.A.
}

\begin{abstract}
Aim: To report on the unique, innovative management of a super morbidly obese patient presenting for fertility preservation in the setting of Grade 2 endometrioid endometrial adenocarcinoma. Case Report: A back-to-back oocyte retrieval and robot-assisted total laparoscopic hysterectomy (RA-TLH) with bilateral salpingo-oophorectomy (BSO) was performed in a 37-year-old nulligravid woman with super morbid obesity $\left(B M I 63 \mathrm{~kg} / \mathrm{m}^{2}\right)$ with endometrial cancer. Main outcome measure: the successful retrieval of oocytes for fertility preservation and oncologic intervention via RA-TLH with BSO in the same operative and anesthetic episode. Results: This combined procedure strategy was successful in our case and is a feasible option for similar high-risk patients in the future. Conclusion: Identifying safe and effective approaches for oocyte retrieval in morbidly obese patients represents a pressing need as obesity rates rise among reproductive-aged women. Back-to-back oocyte retrieval and RA-TLH with BSO are not only feasible, but could also decrease significant anesthetic risks for morbidly obese patients.
\end{abstract}

Endometrial cancer is the fourth most common cancer in American women and the most common malignancy of the lower female genital tract, with an estimated 61,380 new cases in the year $2017(1,2)$. Due in large part to the increasing rate of obesity (BMI $\geq 30 \mathrm{~kg} / \mathrm{m}^{2}$ ), present in $35 \%$ of reproductiveaged women in 2014 (3), the national incidence of endometrial cancer has increased by $2.5 \%$ annually (4). Though endometrial

This article is freely accessible online.

Correspondence to: Esther H. Chung, Center for Infertility and Reproductive Surgery, 75 Francis St., ASB1-3, Boston, MA 02115 , U.S.A. Tel: +1 6177324648, e-mail: esther_chung@hms.harvard.edu

Key Words: Fertility preservation, obesity (BMI), endometrial cancer, anesthesia, oocyte retrieval, robot-assisted laparoscopic hysterectomy. cancer is less common in women under the age of 44 years (with an approximate $9 \%$ incidence in this group), among those with $\mathrm{BMI} \geq 35 \mathrm{~kg} / \mathrm{m}^{2}$, the risk for developing endometrial cancer may be up to 22-fold higher (5). Anovulation due to polycystic ovarian syndrome (PCOS), present in up to a fifth of young women aged 20 to 40 years, leads to unopposed estrogen exposure and represents a major cause of the increased risk of endometrial carcinoma in obese reproductive-age women $(6,7)$.

For a growing number of obese premenopausal women with endometrial cancer, fertility preservation represents a pressing need. For the appropriate patient with grade 1 endometrial cancer on dilation and curettage and no evidence of myometrial invasion or extra-uterine disease on radiographic studies such as MRI, conservative management with hormonal therapy such as progestins is an option for fertility preservation (8). However, up to $30 \%$ of patients present with grade 2 or 3 histology or myometrial invasion, which require definitive surgical management with a total hysterectomy with bilateral salpingooophorectomy (BSO) (5, 9). For women facing definitive surgery for gynecologic cancer, fertility preservation options are largely limited to oocyte and/or embryo cryopreservation for future use in a gestational carrier.

Defined as having a BMI $\geq 40 \mathrm{~kg} / \mathrm{m}^{2}$, morbid obesity (class III) has become the most common chronic disease of the Western world, growing to epidemic proportions in U.S.A. Between 2000 and 2010, the prevalence of morbid obesity increased by $70 \%$ to 1 in 15 adults, while the prevalence of "super morbid obesity" or BMI $\geq 50 \mathrm{~kg} / \mathrm{m}^{2}$ increased even faster (10). Among women requiring pelvic surgery, obesity increases the degree of technical difficulty, morbidity and associated complications, including prolonged hospital stay, and increased risk of infection, venous thrombosis and anesthetic complications (11). Robot-assisted laparoscopy allows for a minimally invasive approach, facilitated by increased dexterity of the robotic arms and the threedimensional optics to improve care and reduce the surgical risk in this patient population. Compared to conventional 
laparoscopy, robot-assisted surgery in obese patients has been associated with shorter hospital stay, and potentially less surgeon fatigue and torque on the abdominal wall (12).

Given obese patients' higher risk of perioperative complications overall, crafting a safe and feasible treatment plan for fertility preservation within the limited time window prior to oncologic intervention requires novel, collaborative, and interdisciplinary solutions. To our knowledge, this is the first report of simultaneous oocyte retrieval and robot-assisted total laparoscopic hysterectomy (RA-TLH) with BSO in a super morbidly obese patient, which was performed safely with excellent oocyte yield.

\section{Case Report}

A 37-year-old nulligravid woman was referred by her Gynecologic Oncologist for discussion of fertility preservation in the setting of a new diagnosis of Grade 2, endometrioid type, endometrial cancer. Her history was notable for morbid obesity with a BMI of $62.8 \mathrm{~kg} / \mathrm{m}^{2}$, PCOS, hypertension well controlled on medications, and hypothyroidism. Prior to her cancer diagnosis, the patient had been undergoing fertility treatment at another center, with completion of three unsuccessful ovulation induction cycles with clomiphene citrate over the past 3 years, of which further details were not available. In the midst of her fourth cycle, an ultrasound demonstrated an endometrial thickness of nearly $15 \mathrm{~mm}$, and subsequent evaluation by endometrial biopsy showed complex hyperplasia. Dilation and curettage was then performed at an outside hospital, revealing Grade 2, endometrioid type endometrial adenocarcinoma. These slides were also reviewed and the diagnosis was confirmed by gynecologic pathologists at our institution. Given the grade of her endometrial cancer, the patient was not a candidate for conservative treatment with progestins, and she was counseled for definitive surgery (8). Given her very high BMI, a RA-TLH with BSO was deemed the most appropriate (12).

Written permission from the patient for publication of this report was obtained.

The patient's oncologic surgery was scheduled for three weeks from her initial Reproductive Endocrinology consultation. Following extensive counseling, she elected to proceed with embryo cryopreservation. The patient's morbid obesity necessitated extensive multidisciplinary preoperative planning involving Gynecologic Oncology, Reproductive Endocrinology, Embryology and Anesthesiology to determine the optimal means of oocyte retrieval. Her high BMI and associated risk of airway obstruction precluded an ambulatory procedure with intravenous sedation, which is the standard of care for oocyte retrievals at our center. As a result, decision was made to perform the oocyte retrieval and hysterectomy in a single coordinated procedure in our main operating rooms (OR). Performing back-to-back procedures would limit the risks associated with two separate anesthetic episodes.
As the patient's ovaries were adequately visualized on her baseline transvaginal ultrasound (TVUS), the decision was made to perform a transvaginal oocyte retrieval in the main OR. The Embryology team would be present in the OR with a microscope, mobile incubator, and all necessary supplies to identify oocytes, which would then be transported to the Embryology laboratory for insemination.

In the event that the patient's ovaries could not be visualized transvaginally, the Gynecologic Oncologist had planned to perform a laparoscopic BSO first and remove them individually through a mini-laparotomy so as to not disrupt the follicles. The ovaries would be placed in sterile media and transported to the Embryology laboratory for ex vivo oocyte retrieval. This plan was not selected as the firstline approach as the laparotomy incision required to remove the stimulated ovaries atraumatically would be much larger than any of the planned laparoscopic incisions.

The patient's antimullerian hormone (AMH) level was 1.0 $\mathrm{ng} / \mathrm{ml}$, with an antral follicle count of 20 . Given her longstanding anovulation, the stimulation was started without preceding withdrawal bleed, two weeks prior to the planned surgery date. The patient was counseled that her follicular development might not perfectly coincide with her surgery, but that retrieval would proceed regardless. An antagonist protocol was selected, using 300 units of Gonal-F ${ }^{\circledR}$ (EMD Serono, Rockland, MA, USA) and 4 vials of Menopur ${ }^{\circledR}$ (Ferring Pharmaceuticals, Parsippany, NJ, USA) per day. On day 14 of stimulation (2 days prior to her hysterectomy), monitoring revealed two lead follicles of $18 \mathrm{~mm}$ in mean diameter by TVUS and an estradiol of $2,479 \mathrm{pg} / \mathrm{ml}$. Final oocyte maturation was triggered with 10,000 units of intramuscular human chorionic gonadotropin that evening (as the risk of ovarian hyperstimulation would be eliminated by oophorectomy), in anticipation of her scheduled combined procedure 36 hours later. Per protocol, the patient had a positive urinary pregnancy test the following day.

\section{Results}

On the morning of surgery, the Reproductive Endocrine and Embryology teams prepared tables in the OR for retrieval and oocyte identification, respectively (Figure 1). General anesthesia was induced; the patient was intubated and placed in the dorsallithotomy position. Antibiotic prophylaxis was provided with intravenous cefotetan per hospital protocol. Sterile drapes were placed, and the vagina was prepared with sterile saline per protocol. Firm, constant pressure was applied both vaginally with the TVUS probe and abdominally by an assistant, in order to visualize the ovaries adequately. With a sterile TVUS probe with needle guide, follicles were aspirated into sterile $5 \mathrm{ml}$ tubes using a 17-gauge needle under continuous negative pressure of $90 \mathrm{~mm} \mathrm{Hg}$. The retrieval process took approximately 45 minutes. A total of 17 oocytes were retrieved and transported to the Embryology laboratory in a warmed travel incubator. 

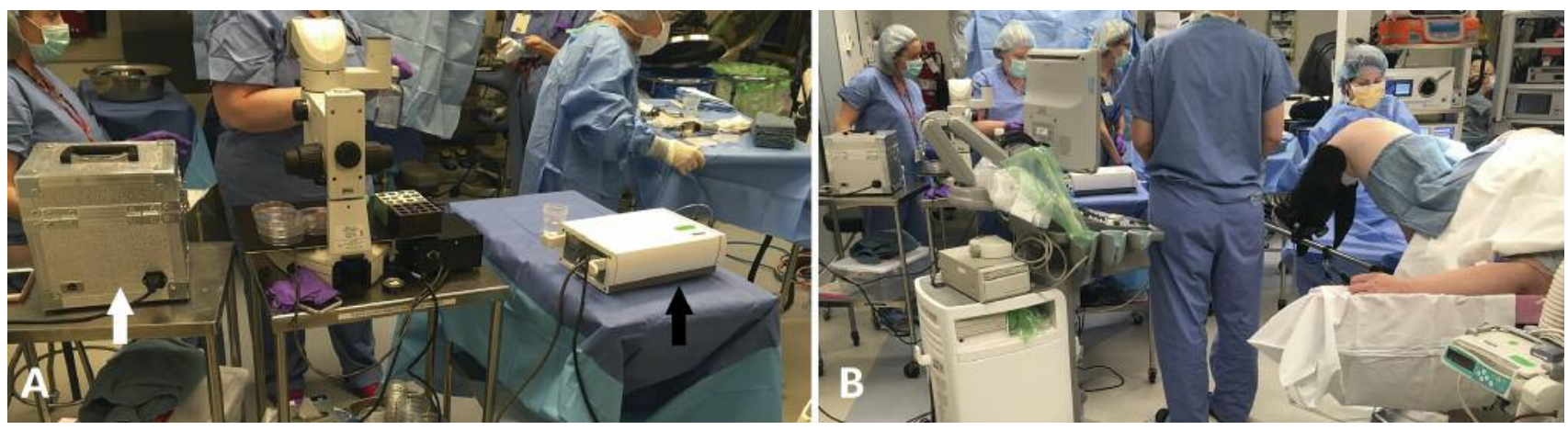

Figure 1. Set-up for transvaginal ultrasound-guided oocyte retrieval by the Embryology (A) and Reproductive Endocrinology (B) teams in the operating room. The mobile incubator is indicated with the white arrow. The aspiration pump for oocyte retrieval is indicated with the black arrow.

After completion of the oocyte retrieval, the patient was prepared with povidone-iodine over the mons and vulva and with chlorhexidine over the abdomen. The gynecologic oncologists then proceeded with the RA-TLH with BSO. Upon laparoscopic entry into the abdomen, the ovaries were prominent with less than $50 \mathrm{ml}$ of intra-abdominal blood and no active bleeding (Figure 2). After the ports were placed, the robot was docked and an uncomplicated hysterectomy with BSO was performed with intact removal of the uterus, cervix, tubes, and ovaries were delivered intact through the vagina (Figure 3). Indocyanine green fluorescence-guided sentinel node biopsy was attempted, but no localization was noted, likely secondary to the patient's habitus. Given her morbid obesity, the patient was admitted overnight; her postoperative course was uncomplicated, and she was discharged home on postoperative day 1 .

Frozen section analysis of the endometrium revealed a focus of noninvasive grade 1 endometrioid adenocarcinoma, obviating the need for nodal staging. Final pathology ultimately revealed a Stage $1 \mathrm{~A}$, grade 1 endometrioid, endometrial adenocarcinoma.

Thirteen of the 17 oocytes retrieved were noted to be mature. On postoperative day 1,9 of the oocytes were found to have fertilized normally and were vitrified, according to protocol, at the pronuclear stage, for future use in a gestational carrier.

\section{Discussion}

Fertility preservation in the setting of endometrial cancer in patients with morbid obesity presents a distinct challenge, due to the increased morbidity of perioperative and anesthetic complications, along with poorer reproductive outcomes including decreased oocyte yield and fertilization rates (13). However, despite these risks, this patient recovered well following her combined surgical procedures, with successful cryopreservation of 9 embryos. It was the combined efforts and effective communication of the

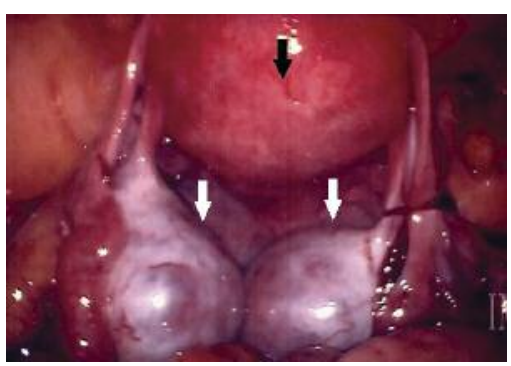

Figure 2. Laparoscopic view of the uterus (black arrow) and stimulated ovaries (white arrows) following oocyte retrieval.

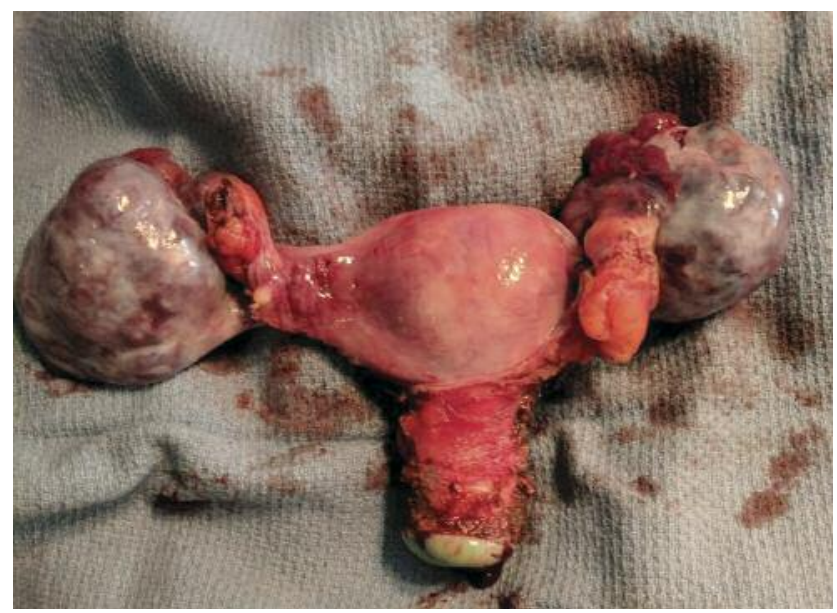

Figure 3. Surgical specimen: Uterus, bilateral tubes and hyperstimulated ovaries following oocyte retrieval.

multidisciplinary care team that enabled a safe, successful strategy for both fertility preservation and the definitive treatment of her malignancy.

No combined procedures of oocyte retrieval and laparoscopic hysterectomy have been previously reported. This 
patient's super morbid obesity provides an added level of complexity to this clinical scenario. Multiple studies attest to the difficulties associated with each procedure alone (12-16). Intra- and post-operative complications of oocyte retrieval, noted more frequently in obese patients, include oxygen desaturations both during and after surgery, conversions from total IV to general anesthesia with endotracheal intubation, resorting to the transabdominal approach for oocyte retrieval, as well as increased postoperative pain and need for additional analgesia, occurred more frequently in the obese patients (16). For this reason, most ambulatory reproductive endocrinology centers set an upper limit of BMI for which oocyte retrieval will be performed. More broadly, gynecologic surgery in obese patients is associated with increased risk of surgical site infection, venous thromboembolism, and wound complications (11). Equally important, anesthetic concerns in obese patients largely involve their compromised respiratory physiology. Particularly in robot-assisted laparoscopic gynecologic surgeries, steep Trendelenburg positioning along with peritoneal $\mathrm{CO} 2$ insufflation leads to decreased lung volumes and increased venous return. This can lead to severe hypercarbia, respiratory acidosis, hypoventilation-related hypoxia and severe head and neck edema (15).

This case report describes a unique and innovative approach to mitigate the significant surgical and anesthetic risks in the morbidly obese patient. Furthermore, this report demonstrates the feasibility of back-to-back oocyte retrieval and RA-TLH with BSO in the same OR and anesthetic episode. Furthermore, in the care of complex patients, anticipation of difficulties and complications is paramount. As such, our team was prepared for the ovaries to be inaccessible transvaginally due to the patient's extreme obesity, which would have required conversion to ex vivo oocyte retrieval from the hyperstimulated ovaries following removal by Gynecologic Oncology.

As the prevalence of obesity in women of childbearing potential continues to increase in the United States of America and worldwide, gynecologists are ever more likely to encounter a morbidly obese reproductive-age patient with endometrial cancer who hopes to preserve her fertility. This novel approach of combined oocyte retrieval and RA-TLH with BSO provided safe and effective reproductive and oncologic care in a highrisk patient. This case demonstrates the importance of multidisciplinary planning in the care of such a complex patient, and the ultimate feasibility of fertility preservation even in the setting of super morbid obesity.

\section{Conflicts of Interest}

The Authors report no conflicts of interest.

\section{Acknowledgements}

No financial support, funding or services were obtained for this study.

\section{References}

1 Purdie DM and Green AC: Epidemiology of endometrial cancer. Best Pract Res Clin Obstet Gynaecol 15: 341-354, 2001.

2 Surveillance, Epidemiology and ERP: Cancer of the Endometrium - Cancer Stat Facts. NIH Natl Cancer Inst, 2017. Available from: https://seer.cancer.gov/statfacts/html/corp.html [last accessed July 30, 2017].

3 Ogden CL, Carroll MD, Fryar CD and Flegal KM: Prevalence of Obesity Among Adults and Youth: United States, 2011-2014. NCHS Data Brief: 1-8, 2015.

4 Constantine G, Kessler G and Goldstein SR: Increased Incidence of Endometrial Cancer Following the WHI. Obstet Gynecol 129: 7S, 2017.

5 Committee on Gynecologic Practice: Committee Opinion No. 619. Obstet Gynecol 125: 274-278, 2015.

6 Salha O, Martin-Hirsch P, Lane G and Sharma V: Endometrial carcinoma in a young patient with polycystic ovarian syndrome: first suspected at time of embryo transfer. Hum Reprod 12: 959962, 1997.

7 Jayakrishnan K, Anupama R, Koshy A and Raju R: Endometrial carcinoma in a young subfertile woman with polycystic ovarian syndrome. J Hum Reprod Sci 3: 38-41, 2010.

8 Burke WM, Orr J, Leitao M, Salom E, Gehrig P, Olawaiye AB, Brewer M, Boruta D, Herzog TJ, Shahin FA and Burke WM: Endometrial cancer: A review and current management strategies: Part II SGO Clinical Practice Endometrial Cancer Working Group for the Society of Gynecologic Oncology Clinical Practice Committee. Gynecol Oncol 134: 393-402, 2014.

9 Denschlag D, Ulrich U and Emons G: The diagnosis and treatment of endometrial cancer: progress and controversies. Dtsch Arztebl Int 108: 571-577, 2010.

10 Sturm R and Hattori A: Morbid obesity rates continue to rise rapidly in the United States. Int J Obes (Lond) 37: 889-891, 2013.

11 Committee on Gynecologic Practice. Committee Opinion No. 619. Obstet Gynecol [Internet]. 2015 Jan [cited 2017 Aug 11];125: 274278. Available from: http://www.ncbi.nlm.nih.gov/ pubmed/25560144

12 Scheib SA, Tanner E, Green IC and Fader AN: Laparoscopy in the morbidly obese: physiologic considerations and surgical techniques to optimize success. J Minim Invasive Gynecol 21: 182-195, 2014.

13 Pandey S, Pandey S, Maheshwari A and Bhattacharya S: The impact of female obesity on the outcome of fertility treatment. J Hum Reprod Sci 3: 62-77, 2010.

14 Ioscovich A, Eldar-Geva T, Weitman M, Altarescu G, Rivilis A and Elstein D: Anesthetic management for oocyte retrieval: An exploratory analysis comparing outcome in in vitro fertilization cycles with and without pre-implantation genetic diagnosis. J Hum Reprod Sci 6: 263-266, 2013.

15 Srivastava A and Niranjan A: Secrets of safe laparoscopic surgery: Anaesthetic and surgical considerations. J Minim Access Surg 6: 91-94, 2010.

16 Egan B, Racowsky C, Hornstein MD, Martin R and Tsen LC: Anesthetic impact of body mass index in patients undergoing assisted reproductive technologies. J Clin Anesth 20: 356-363, 2008. 